\title{
Scale invariance and related properties of $q$-Gaussian systems
}

\author{
C. Vignat ${ }^{\mathrm{a}}$, A. Plastino ${ }^{\mathrm{b}, *}$ \\ ${ }^{a}$ L.T.H.I., E.P.F.L, Lausanne, Switzerland \\ ${ }^{\mathrm{b}}$ La Plata National University, Exact Sciences Faculty \& National Research Council (CONICET), C.C. 727, 1900 La Plata, Argentina
}

Received 29 November 2006; received in revised form 29 January 2007; accepted 2 February 2007

Available online 8 February 2007

Communicated by A.R. Bishop

\begin{abstract}
We advance scale-invariance arguments for systems that are governed (or approximated) by a $q$-Gaussian distribution, i.e., a power law distribution with exponent $Q=1 /(1-q) ; q \in \mathbb{R}$. The ensuing line of reasoning is then compared with that applying for Gaussian distributions, with emphasis on dimensional considerations. In particular, a Gaussian system may be part of a larger system that is not Gaussian, but, if the larger system is spherically invariant, then it is necessarily Gaussian again. We show that this result extends to $q$-Gaussian systems via elliptic invariance. The problem of estimating the appropriate value for the Tsallis' parameter $q$ is revisited. A kinetic application is also provided.
\end{abstract}

(c) 2007 Elsevier B.V. All rights reserved.

PACS: 05.40.-a; 05.20.Gg; 02.50.-r

Keywords: Scale invariance; Elliptical invariance; $q$-Gaussian distributions; Super-statistics

\section{Introduction and background material}

Homogeneous power-laws, such as Newton's universal law of gravitational attraction, for instance, abound in nature. They are, by definition, self-similar and thus true in all scales. Systems statistically described by power-law probability distributions are rather ubiquitous [1] and thus of perennial interest [2]. In this Letter we wish to give careful scale-invariance consideration to systems that are governed (or described) by a special kind of power-law probability distribution functions (PDF), namely, the $q$-Gaussian function. Consider a system $\mathcal{S}$ described by a vector $X$ with $n$ components. We say that $X$ is $q$-Gaussian distributed if its probability distribution function writes as described by (1.6)-(1.7) below.

It is well known that for such $\mathcal{S}$-systems one can appeal to Jaynes' maximum entropy principle (MaxEnt) [3] under a $q$-covariance constraint with a generalized (or $q$-) information

\footnotetext{
* Corresponding author.

E-mail address: plastino@fisica.unlp.edu.ar (A. Plastino).
}

measure

$$
H_{q}(x)=\frac{1}{1-q} \int_{\mathbb{R}^{n}} d x\left[f^{q}(x)-f(x)\right] ; \quad q \in \mathbb{R},
$$

as the protagonist [4]. This measure has been found to be useful in extracting information pertaining to systems that are characterized by either (1) fractal nature, (2) long-memory, or (3) long-range interactions [5]. Employing $H_{q}$ for other types of system has generated controversy [6] which is of no relevance to our present purposes. We will focus attention upon properties of Gaussian systems that remain valid for their $q$ Gaussian counterparts as well (as $q$ becomes different from unity), with emphasis on the dimensional properties of both kinds of systems. It is well known that if a system "is Gaussian", any part (subsystem) of it is still Gaussian. This property holds for $q$-Gaussian systems as well, as proved in [7]. A more interesting result is the inverse phenomenon: a Gaussian system may be part of a larger system that is not Gaussian. However, if the larger system is spherically invariant, then it is necessarily Gaussian again. Surprisingly enough, this "inverse property" has gone largely ignored in the statistical literature. In this work we will not only provide a simple proof for it but we will 
show that it can be extended to $q$-Gaussian distributions as well. These results can be given a physical interpretation within the framework of the estimation of the parameter $q$ of a given system [5]. We will prove that, if spherical symmetry prevails, such estimation can be performed using only a restricted, observable part of the system and that the overall parameter $q$ for the entire system can be retrieved provided the dimension of the system is known. We begin our considerations by introducing the two basic notions, namely spherical symmetry and $q$-Gaussian systems. We will also apply our results to a simple case of kinetic theory via the Beck-Cohen superstatistics theory [8-14].

\subsection{Spherical symmetry}

A really momentous symmetry is that of invariance against rotations. It is found in the fundamental laws of nature and constitutes one of the most powerful principles in elucidating the structure of individual atoms, complicated molecules, and entire crystals. Also, it characterizes the shape of many systems. We can cite self-gravitating systems like stars and planets, that have quasi-spherical shape if their mass is large enough. Also, many atomic nuclei are spherical, and many molecules as well, etc. Conservation of angular momentum, a very frequent occurrence, is a result of the isotropy of space itself [15]. We discuss now some properties of spherical probability distributions.

The characteristic function associated with a random vector $X \in \mathbb{R}^{n}$ is

$\varphi_{X}(U)=E e^{i U^{t} X} ; \quad U \in \mathbb{R}^{n}$.

Under the hypothesis, discussed for instance in the textbook [16, XV.3], that $\varphi_{X} \in \mathcal{L}_{1}\left(\mathbb{R}^{n}\right)$, there is a one-to-one relation between $\varphi_{X}$ and the probability density function $f_{X}$ of $X$. The random vector $X$ is said to have a spherical distribution if its characteristic function $\varphi_{X}$ satisfies

$\varphi_{X}(U)=\phi(\|U\|)$

for some scalar function $\phi: \mathbb{R}^{+} \rightarrow \mathbb{R}$ which is then called the characteristic generator of the spherical distribution. We will write $X \sim \mathcal{S}_{n}(\phi)$ in this case. It is well known that an equivalent definition for a spherical random vector $X$ is

$X \sim A X ; \quad \forall A$ orthogonal,

where $\sim$ denotes equality in distribution.

Spherical random vectors have, as it is well known, the following properties:

(1) All marginal distributions of a spherical distributed random vector are spherical.

(2) All marginal characteristic functions have the same characteristic generator.

(3) If $X \sim \mathcal{S}_{n}(\phi)$ then

$X \sim r T_{n}$,

where $T_{n}$ is a random vector distributed uniformly on the unit sphere surface in $\mathbb{R}^{n}$ and $r$ is a positive random variable independent of $T_{n}$.
Let us remark that a spherically distributed random vector does not necessarily possess a density.

A generalization of the concept of spherical distribution is given by elliptical distributions, to which the multi-normal distribution belongs. Elliptical distributions have recently gained a lot of attention in financial mathematics, being of use particularly in risk management. An $n$-random vector $Y$ is said to have an elliptical distribution with so-called characteristic matrix $C_{Y}(n \times n)$ if $Y \sim A X$, where $X \sim \mathcal{S}_{n}(\phi)$ and $A$ is an $n \times n$ deterministic matrix such that $A^{t} A=C_{Y}$ and $\operatorname{rank}\left(C_{Y}\right)=n$. We shall write $Y \sim \mathcal{E}_{n}\left(C_{Y}, \phi\right)$.

\section{2. q-Gaussian systems}

An $n$-components vector $X$ is $q$-Gaussian distributed if its distribution $f_{X}$ maximizes the $q$-information (1.1) under a generalized covariance constraint

$\int_{\mathbb{R}^{n}} d x x x^{t} f^{q}(x)=K$

where $K$ is a positive definite matrix.

A classical result is that this PDF writes as follows [2]:

- in the case $1<q<\frac{n+2}{n}$

$$
\begin{aligned}
& f_{X}(X) \\
& \quad=A_{q}\left(1+\frac{q-1}{(n+4)-q(n+2)} X^{t} K^{-1} X\right)^{\frac{1}{1-q}} .
\end{aligned}
$$

In the following, we will use matrix $\Lambda$ related to the covariance matrix $K=E X X^{t}$ in the fashion [17]

$\Lambda=m K$,

where the number of degrees of freedom is defined as [17]

$m=\frac{2}{q-1}-n$.

In this way, the quadratic form in (1.6) writes simply $X^{t} \Lambda^{-1} X$. However, form (1.6) reveals more clearly that, as $q \rightarrow 1^{+}$, the $q$-Gaussian distribution reduces to the classical Gaussian distribution.

We note that the condition $1<q<\frac{n+2}{n}$ (equivalently $m>0$ ) ensures convergence of integrals $\int_{\mathbb{R}^{n}} d x f_{X}$, $\int_{\mathbb{R}^{n}} d x x x^{t} f^{q}$ and $\int_{\mathbb{R}^{n}} d x f^{q}$ while for $\frac{n+2}{n}<q<\frac{n}{n-2}$, only the latest integral converges.

Moreover, the partition function $Z_{q}=1 / A_{q}$ reads [17]

$Z_{q}=\frac{\Gamma\left(\frac{1}{q-1}-\frac{n}{2}\right)|\pi \Lambda|^{1 / 2}}{\Gamma\left(\frac{1}{q-1}\right)}$

and the characteristic function is

$\varphi_{X}(U)=\frac{2^{1-\frac{m}{2}}}{\Gamma\left(\frac{m}{2}\right)} z^{\frac{m}{2}} K_{\frac{m}{2}}(z)$

with $z=\sqrt{U^{t} \Lambda U}$ and $K$ is the modified Bessel function of the second kind. 
- in the case $q<1$

$$
\begin{aligned}
& f_{X}(X) \\
& \quad=A_{q}\left(1-\frac{1-q}{(n+4)-q(n+2)} X^{t} K^{-1} X\right)_{+}^{\frac{1}{1-q}} .
\end{aligned}
$$

Defining matrix $\Sigma=d K$ and parameter $d$ as

$d=\frac{2}{1-q}+n$,

the quadratic form in (1.10) simplifies to $X^{t} \Sigma^{-1} X$, but form (1.10) shows again clearly the fact that as $q \rightarrow 1^{-}$, the $q$-Gaussian density converges to the Gaussian density. The partition function is

$$
Z_{q}=\frac{\Gamma\left(\frac{2-q}{1-q}\right)|\pi \Sigma|^{1 / 2}}{\Gamma\left(\frac{2-q}{q-1}+\frac{n}{2}\right)}
$$

and the characteristic function is

$$
\varphi_{X}(U)=2^{\frac{d}{2}-1} \Gamma\left(\frac{d}{2}\right) \frac{J_{\frac{d}{2}-1}(z)}{z^{\frac{d}{2}-1}}
$$

where $z=\sqrt{U^{t} \Sigma U}$ and $J$ is the Bessel function of the first kind.

We begin in the next section to advance our present results.

\section{Size behavior of the $q$-parameter}

Our first result revolves around the behavior of the nonextensivity parameter $q$ as a function of the dimension of the system and is embodied in the following theorem, the proof of which is given in Appendix A.

Theorem 1. Assume that a system $X_{n} \in \mathbb{R}^{n}$ follows a $q$ Gaussian distribution with parameter $q_{n}$; then with $1 \leqslant k \leqslant$ $n-1$, any $k$-dimensional subsystem $X_{k}=\left[x_{1}, \ldots, x_{k}\right]^{t}$ of $X_{n}$ is $q$-Gaussian distributed with parameter

$q_{k}=1-\frac{2\left(1-q_{n}\right)}{2+(n-k)\left(1-q_{n}\right)}$

what can be equivalently written as

$\frac{2}{1-q_{k}}=\frac{2}{1-q_{n}}+n-k$.

Reciprocally, assume that an $n$-dimensional and spherical system $X_{n}$ contains a $k$-dimensional subsystem $X_{k}$ that follows a $q$-Gaussian distribution with parameter $q_{k}$; then system $X_{n}$ is itself $q$-Gaussian distributed with parameter $q_{n}$ defined as in (2.1).

Recall that for any $n$-dimensional orthogonal transformation $A$, there exists an orthogonal decomposition of $\mathbb{R}^{n}$ as

$\mathbb{R}^{n}=E \oplus F \oplus G_{1} \oplus \cdots \oplus G_{k}$

into stable subspaces, the restriction of $A$ to each subspace being:
- the identity transformation for subspace $E$,

- minus the identity transformation for subspace $F$,

- a two-dimensional planar rotation of angle $\theta_{k}$ for subspace $G_{k}$.

Moreover, $n$-dimensional $q$-Gaussian distributions with parameter $q_{n}$ arise in statistical physics as the canonical distributions of systems with maximal $q$-entropy $H_{q}$ of order $q_{n}$ and fixed covariance matrix. The result of Theorem 1 can be paraphrased in this way: if a system of dimension $k$ has maximum $q$-entropy of order $q_{k}$, and is also part of a spherical system of dimension $n>k$, then the whole system maximizes the $q$ entropy with parameter $q_{n}$ related to $q_{k}$ as in (2.1). Notice that in (2.1), as $q_{k} \rightarrow 1$ then $q_{n} \rightarrow 1$ and we deduce that if a spherical system has a Gaussian part, then it is necessarily Gaussian as well.

We note also that $q_{n}<1$ implies $q_{k}<1$ while $q_{n}>1 \mathrm{im}$ plies $q_{k}>1$. This result is natural since cases $q>1$ and $q<1$ correspond to two different types of distributions: according to Beck and Cohen's superstatistics principle [8-14]:

- $q>1$ that corresponds to a Gaussian system subjected to fluctuations that are independent of the state of the system.

- $q<1$ that corresponds again to a fluctuating Gaussian system for which the amplitude of the fluctuations depends on the system's state.

Alternatively, these two cases can be characterized as follows. Our system is here described by the random vector $X$ : if $q>1$, then $X$ has unbounded support, contrarily to the bounded support associated to the case $q<1$ [17].

A last remark at this step concerns Eqs. (2.1) and (2.2): in the case $q>1$, these equations express the fact that the number of degrees of freedom $m$ is invariant by marginalization. This is again in accordance with the superstatistics theory, since this parameter $m$ characterizes the strength of the temperature fluctuations to which the $n$-dimensional Gaussian system is submitted: any subsystem of this Gaussian system is in turn submitted to the same fluctuations. We note that Eqs. (2.1) and (2.2) appear for example in Refs. [18,19]. The first of these two papers refers, in the context of a generalization of the central limit theorem, to the definition of the $q$-Fourier transform: a link between such context and the present one remains to be established.

For the other case, namely, $q<1$, Eqs. (2.1) and (2.2) express the invariance of parameter $d$ defined by (1.11) by marginalization: for a geometric interpretation and reasons of invariance of this parameter, the reader is referred to [17].

Thus, we obtain the following rather natural result: reduction or enlargement of a $q$-Gaussian system does not change its superstatistical nature.

\section{A second result: Multi-component systems}

\subsection{Average behavior}

It may happen that measuring the behavior of only one component $x_{1}$ of a large system $X \in \mathbb{R}^{n}$ is not physically feasible, 
and one has to content oneself with measuring instead the behavior of a superposition of contributions from (or average of) several components [20], in the form

$\langle X\rangle=\sum_{i=1}^{n} a_{i} x_{i}$,

where the deterministic coefficients $a_{i} \in \mathbb{R}$ characterize the measurement device. The following theorem (see Ref. [21]) allows to give a special characterization of this average value in the case of spherical systems.

Theorem 2. (See [21, Theorem 2.4].) If $X \in \mathbb{R}^{n}$ is spherically distributed and $A=\left(a_{1}, \ldots, a_{n}\right)^{t}$ is a deterministic vector then

$\langle X\rangle=\sum_{i=1}^{n} a_{i} x_{i}$

is distributed as $\|A\| x_{1}$, where $\|A\|$ is the Euclidean norm of $A$.

In the next subsection we extract rather interesting physical conclusions from this theorem.

\subsection{Application to the estimation of $q$}

An important problem in non-extensive statistics is the estimation of the non-extensivity parameter $q_{n}$ associated to an $n$-dimensional system that follows a $q$-Gaussian distribution [5]. We provide here some hints about a possible estimation strategy in the case $q>1$, assuming that we have access to averaged measures of the system of the type (3.1).

Assuming $q_{n}>1$, then if $X_{n}$ follows distribution (1.6), the averaged measure $\langle X\rangle$ is distributed as

$f_{\langle X\rangle}(x)=\frac{A_{q_{1}}}{\|A\|}\left(1+\frac{x^{2}}{\lambda\|A\|^{2}}\right)^{-\frac{m+1}{2}}$

with $m=2 /\left(q_{1}-1\right)-1$ and $\lambda=\Lambda_{1,1}$. As a consequence, a possible estimation strategy of parameter $q_{n}$ follows the three following steps:

(1) since the "tail-behavior" of the distribution of $f_{\langle X\rangle}$ is

$f_{\langle X\rangle}(x) \sim x^{-(m+1)}$

(where $\sim$ means here asymptotic equivalence), parameter $m$ can be estimated as the Lévy exponent of the distribution of the average measure of the system, ${ }^{1}$

(2) the non-extensivity parameter $q_{1}$ of $\langle X\rangle$ can be computed using (1.8) as

$q_{1}=\frac{m+3}{m+1}$,

(3) the non-extensivity parameter $q_{n}$ of the $n$-dimensional system $X_{n}$ can in turn be deduced using (2.1) as

$q_{n}=\frac{2-(n+1)\left(1-q_{1}\right)}{2-(n-1)\left(1-q_{1}\right)}$.

\footnotetext{
${ }^{1}$ Note that there exist other statistical approaches to the estimation of parameter $q_{1}$ : see [26].
}

As a new result we find that if the dimension $n$ of the system is known, its non-extensivity parameter $q_{n}>1$ can be evaluated from any measurement of the type (3.1).

\subsection{A kinetic application}

\subsubsection{Theoretical framework}

Another application of the latter result can be provided in the context of the kinematics of collision events. We envision a scenario in which attention is focused on the particles of a system interacting with a heat bath (a fundamental problem in thermodynamics). An elastic collision between (i) a system's particle with momentum $P$, mass $M$, velocity $V$, and energy $E$ and (ii) a particle from the heat bath with momentum $p$, mass $m$, velocity $v$, and energy $\epsilon$, verifies [22]

$E+\epsilon=\hat{E}+\hat{\epsilon}$,

$P+p=\hat{P}+\hat{p}$

where "hats" refer to quantities after the collision. In the nonrelativistic case, these quantities write

$P=M V, \quad p=m v$,

$E=\frac{\|P\|^{2}}{2 M}, \quad \epsilon=\frac{\|p\|^{2}}{2 m}$,

where momenta are 3-dimensional quantities. These equations can be solved as

$\hat{P}(p, P)=\left(\frac{2 M}{M+m}\right) p+\left(\frac{M-m}{M+m}\right) P$,

$\hat{p}(p, P)=\left(\frac{m-M}{M+m}\right) p+\left(\frac{2 m}{M+m}\right) P$.

Assuming that $P$ and $p$ are independent random variables, we look for stationary distributions for $p$ and $P$, that is, for probability density functions $f_{p}$ and $f_{P}$ such that if $p \sim f_{p}$ and $P \sim f_{P}$ then after the collision, $\hat{p} \sim f_{p}$ and $\hat{P} \sim f_{P}$. An obvious pair of stationary solutions is given [22] by the independent Maxwell solutions

$f_{P}(P)=\frac{1}{\left(2 \pi M k_{B} T\right)^{3 / 2}} \exp \left(-\frac{\|P\|^{2}}{2 M k_{B} T}\right)$,

$f_{p}(p)=\frac{1}{\left(2 \pi m k_{B} T\right)^{3 / 2}} \exp \left(-\frac{\|p\|^{2}}{2 m k_{B} T}\right)$.

\subsubsection{The correlated scenario}

Suppose however that the assumption of independence between momenta $p$ and $P$ does not hold. Such is the case, for example, when the corresponding particles are subject to the same fluctuations (an interpretation for this scenario is provided in the following subsection). In this instance we look for a stationary joint distribution for $p$ and $P$, i.e., for a probability density function $f_{p, P}$ such that if $(p, P) \sim f_{p, P}$ then, after the collision, $(\hat{p}, \hat{P}) \sim f_{p, P}$ again. We note that this in turn implies $\hat{p} \sim f_{p}$ and $\hat{P} \sim f_{P}$.

We need here an extension of Theorem 2 as given below, the proof of which can be found, for example, in [23]. 
Proposition 3. If $X \sim \mathcal{E}_{n}\left(C_{X}, \phi\right)$ and $A$ is a full-rank $(n \times n)$ matrix then $Y=A X \sim \mathcal{E}_{n}\left(C_{Y}, \phi\right)$ with

$C_{Y}=A C_{X} A^{t}$.

Now assume that

$C_{X}=\left[\begin{array}{cc}m I_{3} & 0_{3} \\ 0_{3} & M I_{3}\end{array}\right]$

$A=\frac{1}{m+M}\left[\begin{array}{cc}(m-M) I_{3} & (2 m) I_{3} \\ (2 M) I_{3} & (M-m) I_{3}\end{array}\right]$,

where $0_{3}$ denotes the $(3 \times 3)$ null matrix and $I_{3}$ the $(3 \times 3)$ identity matrix. Then

$C_{Y}=A C_{X} A^{t}=C_{X}$.

We are now in a position to deduce the following result:

Theorem 4. If $\left(\begin{array}{l}p \\ P\end{array}\right) \sim \mathcal{E}_{6}\left(C_{X}, \phi\right)$ with characteristic matrix $C_{X}$ as above, then the momenta vector after the collision $\left(\begin{array}{l}\hat{p} \\ \hat{p}\end{array}\right) \sim$ $\mathcal{E}_{6}\left(C_{X}, \phi\right)$. As a consequence, any elliptical joint distribution with characteristic matrix $C_{X}$ is stationary. In particular, $p$ and $\hat{p}$ have the same distribution, as well as $P$ and $\hat{P}$.

\subsubsection{Superstatistics at work}

A more physical interpretation can be given to the preceding result, using the notion of superstatistics [8]. We know from [22] that a pair of independent Gaussian momenta are stationary for the collision process. Now,

- if $p \sim \mathcal{N}_{3}\left(m k_{B} T\right)$ (the 3-dimensional Gaussian distribution with covariance matrix $\left.m k_{B} T I_{3}\right)$, and

- if $P \sim \mathcal{N}_{3}\left(M k_{B} T\right)$, then

- $\hat{p} \sim \mathcal{N}_{3}\left(m k_{B} T\right)$ and $\hat{P} \sim \mathcal{N}_{3}\left(M k_{B} T\right)$.

Since

$\hat{P}(p, P)=\left(\frac{2 M}{M+m}\right) p+\left(\frac{M-m}{M+m}\right) P$,

choosing any (dimensionless) random variable $a$ independent of both $p$ and $P$, and defining the new quantities $q=a p, Q=$ $a P$, and $\hat{Q}=a \hat{P}$, we deduce that

$\hat{Q}(q, Q)=\left(\frac{2 M}{M+m}\right) q+\left(\frac{M-m}{M+m}\right) Q$,

so that, obviously, the pair $(q, Q)$ is another couple of momenta whose distribution is stationary. Obviously, variables $q=a p$ and $Q=a P$ are not independent ones, since they share the same random scale factor $a$ (unless $a$ is almost surely a constant, which reduces to the Gaussian case).

As a special case, if $a$ follows an inverse chi-distribution with $m$ degrees of freedom, one immediately finds [8] that the random vector $X=\left(\begin{array}{l}p \\ P\end{array}\right)$ follows a Tsallis-distribution

$f_{X}(X)=A_{q}\left(1+\frac{q-1}{(n+4)-q(n+2)} X^{t} C_{X}^{-1} X\right)^{\frac{1}{1-q}}$,

with non-extensivity index $q>1$ related to parameter $m$ as in formula (1.8) with $n=6$. We remark that the random variable $a$ can be interpreted, in such a context, as representing temperature's fluctuations, as shown by Beck and Cohen [8]. Thus, the presence of temperature fluctuations indicates that the momenta of the incoming colliding particles are correlated. Conversely, if they are correlated, then temperature fluctuations ensue. This scenario is a feasible one if the heat bath is a finite one, which, in turn, establishes a natural connection with an old result of Plastino and Plastino [24].

\section{Conclusions}

In this work we have considered physical applications of a largely ignored result of the statistical literature: a Gaussian system may be part of a larger system that is not Gaussian. However, if this larger system is spherically invariant, then it is necessarily Gaussian again.

We have provided a simple proof for it and we have shown that it can be extended to $q$-Gaussian distributions as well. Our results have been given a physical interpretation within the framework of the problem of estimation of the $q$-Gaussian parameter $q$. Also, we applied them to a simple instance of kinetic theory involving Beck and Cohen superstatistics [8].

\section{Appendix A. Proofs}

\section{A.1. Proof of Theorem 1}

We give here a simple proof of Theorem 1, the principle of which has been kindly suggested to us by Pr. Wlodek Bryc and Pr. Jacek Wesolowski. Assuming first that $X_{k}=\left[x_{1}, \ldots, x_{k}\right]^{t}$ is $q$-Gaussian with parameter $q_{k}>1$, we deduce that, with $U_{k}=$ $\left[u_{1}, \ldots, u_{k}\right]^{t}$,

$$
\begin{aligned}
\varphi_{X_{k}}\left(U_{k}\right) & =\phi\left(\left\|U_{k}\right\|\right) \\
& =\frac{2^{1-\frac{m}{2}}}{\Gamma\left(\frac{m}{2}\right)}\left\|U_{k}\right\|^{\frac{m}{2}} K_{\frac{m}{2}}\left(\left\|U_{k}\right\|\right)
\end{aligned}
$$

so that

$\phi(u)=\frac{2^{1-\frac{m}{2}}}{\Gamma\left(\frac{m}{2}\right)}\|u\|^{\frac{m}{2}} K_{\frac{m}{2}}(\|u\|)$

and

$$
\begin{aligned}
\varphi_{X_{n}}\left(U_{n}\right) & =\phi\left(\left\|U_{n}\right\|\right) \\
& =\frac{2^{1-\frac{m}{2}}}{\Gamma\left(\frac{m}{2}\right)}\left\|U_{n}\right\|^{\frac{m}{2}} K_{\frac{m}{2}}\left(\left\|U_{n}\right\|\right)
\end{aligned}
$$

and $X_{n}$ is $q$-Gaussian with dimension $n$ and $m$ degrees of freedom, and thus has non-extensivity parameter $q_{n}$ such that

$m=\frac{2}{q_{k}-1}-k=\frac{2}{q_{n}-1}-n$.

The same result applies with $q_{k}<1$ by considering characteristic function defined as in (1.12). 


\section{A.2. An alternate proof of Theorem 1}

We provide here an alternate proof based on stochastic representations, as first used in [25], extending their result to the case $q>1$. Assume that $X_{k} \in \mathbb{R}^{k}$ is $q$-Gaussian distributed with parameter $q_{k}$ : a stochastic representation of $X_{k}$ is (see [21])

$X_{k}=\frac{\chi_{k}}{\chi_{d}} Z_{k}$

where $\chi_{k}$ and $\chi_{d}$ are independent and chi-distributed random variables, where $d=\frac{2}{q_{k}-1}-k$ and $Z_{k}$ is uniform on the sphere. We know moreover that if $X_{n}=r Z_{n}$ then

$X_{k}=r d_{1} Z_{k}$

where $d_{1}^{2} \sim \beta_{\frac{k}{2}, \frac{n-k}{2}}$. We deduce that

$r d_{1} Z_{k}=\frac{\chi_{k}}{\chi_{d}}$

or

$r^{2} d_{1}^{2}=\frac{\chi_{k}^{2}}{\chi_{d}^{2}}$.

But by Luckacs theorem ${ }^{2}$ :

$\frac{\hat{\chi}_{n}^{2}}{\hat{\chi}_{d}^{2}} \frac{\tilde{\chi}_{k}^{2}}{\tilde{\chi}_{k}^{2}+\tilde{\chi}_{n-k}^{2}}=\frac{\chi_{k}^{2}}{\chi_{d}^{2}}$

we deduce that

$r=\frac{\chi_{n}}{\chi_{d}}$

and that $X_{n}=r Z_{n}$ is $q$-Gaussian distributed with parameter $q_{n}=1+\frac{2}{d+n}=1+\frac{2\left(q_{k}-1\right)}{2+(n-k)\left(q_{k}-1\right)}$.

\section{References}

[1] M. Scroeder, Fractals, Chaos, Power Laws, Freeman, NY, 1991.

[2] M. Gell-Mann, C. Tsallis (Eds.), Nonextensive Entropy: Interdisciplinary Applications, Oxford Univ. Press, Oxford, 2004;

V. Latora, A. Rapisarda, C. Tsallis, Physica A 305 (2002) 129, and references therein;

S. Abe, Y. Okamoto (Eds.), Nonextensive Statistical Mechanics and Its Applications, Springer, Berlin, 2001;
C. Tsallis, Braz. J. Phys. 29 (1999) 1;

C. Tsallis, J. Stat. Phys. 52 (1988) 479;

A. Plastino, A.R. Plastino, Braz. J. Phys. 29 (1999) 50.

[3] E.T. Jaynes, Phys. Rev. 106 (1957) 620;

E.T. Jaynes, Phys. Rev. 108 (1957) 171;

R.D. Rosenkrantz (Ed.), Papers on Probability, Statistics and Statistical Physics, Reidel, Dordrecht, 1987;

A. Katz, Principles of Statistical Mechanics, The Information Theory Approach, Freeman, San Francisco, 1967.

[4] C. Vignat, A. Plastino, Physica A 361 (2006) 139L;

C. Vignat, A. Plastino, A.R. Plastino, Nuovo Cimento B 120 (2005) 951.

[5] S. Abe, Y. Okamoto (Eds.), Nonextensive Statistical Mechanics and Its Applications, Springer, Berlin, 2001;

M. Gell-Mann, C. Tsallis (Eds.), Nonextensive Entropy: Interdisciplinary Applications, Oxford Univ. Press, Oxford, 2004, and references therein; G. Kaniadakis, M. Lissia, A. Rapisarda (Eds.), Nonextensive Statistical Mechanics and Physical Applications, Physica A 305 (2002) (Special issue), and references therein.

[6] Europhys. News 36 (2005) (special issue); M. Buchanan, New Scientist 187 (2005) 34.

[7] C. Vignat, A.O. Hero, J.A. Costa, Physica A 340 (2004) 147.

[8] C. Beck, E.G.D. Cohen, Physica A 322 (2003) 267.

[9] C. Beck, Contin. Mech. Thermodyn. 16 (2004) 293.

[10] C. Beck, Phys. Rev. Lett. 87 (2001) 180601.

[11] C. Beck, E.G.D. Cohen, Physica A 344 (2004) 393.

[12] C. Beck, Physica A 342 (2004) 139.

[13] C. Beck, Physica D 193 (2004) 195.

[14] H. Touchette, C. Beck, Phys. Rev. E 71 (2005) 016131.

[15] R.B. Lindsay, H. Margenau, Foundations of Physics, Dover, NY, 1957.

[16] W. Feller, An Introduction to Probability Theory and Its Applications, vol. II, John Wiley and Sons, 1970.

[17] C. Vignat, A. Plastino, Phys. Lett. A 343 (2005) 411.

[18] S. Umarov, S. Steinberg, C. Tsallis, cond-mat/0603593; S. Umarov, S. Steinberg, C. Tsallis, cond-mat/0606040; S. Umarov, S. Steinberg, C. Tsallis, cond-mat/0606038.

[19] R.S. Mendes, C. Tsallis, Phys. Lett. A 285 (2001) 273.

[20] B.R. Frieden, Physics from Fisher Information, Cambridge Univ. Press, Cambridge, England, 1998.

[21] K.T. Fang, S. Kotz, K.W. Ng, Symmetric Multivariate and Related Distributions, Chapman \& Hall, London/New York, 1990.

[22] J. Dunkel, P. Hänggi, cond-mat/0606487.

[23] K.C. Chu, IEEE Trans. Automat. Control 18 (1973) 499.

[24] A.R. Plastino, A. Plastino, Phys. Lett. A 193 (1994) 140.

[25] J.-J. Liang, P.M. Bentler, Statist. Probab. Lett. 40 (1998) 155.

[26] V.E. Bening, V.Yu. Korolev, Theory Probab. Appl. 49 (2005) 377.

[27] N. Tsilevich, A. Vershik, M. Yor, J. Funct. Anal. 185 (2001) 274.

\footnotetext{
2 If $X$ and $Y$ are independent then $\frac{X}{X+Y}$ and $X+Y$ are independent if and only if they have standard Gamma distributions. See [27].
} 